\title{
Study and designed of an active infrared system for in-situ characterization of thermal resistance of building envelopes
}

\author{
by L. Gavérina *, T. Ha**, J. Waeytens ***, V. Feuillet**, J-L. Manceau*,

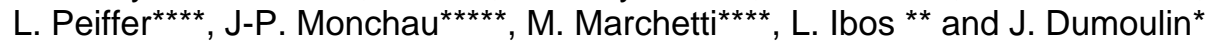 \\ *IFSTTAR, COSYS-SII, Bouguenais, F-44344, France, \& , Inria, I4S Team, Rennes, F-35042, France \\ **UPEC, CERTES, 61 av. Général de Gaulle, Créteil, F-94010, France \\ ***IFSTTAR, COSYS-LISIS, 14-20 Boulevard Newton, Champs-sur-Marne, F-77447, France. \\ ****Cerema - Dter Est - Laboratoire de Nancy, 71 rue de la Grande haie, Tomblaine, F-54510, France \\ *****THEMACS Ingénierie, 23 Rue Alfred Nobel, Champs-sur-Marne, F-77420, France
}

\begin{abstract}
In the research framework of the RESBATI project, we have studied and developed an in-situ thermal resistance estimation protocol by combining active thermal measurements with infrared camera, local temperature and heat flux sensors with inverse models. A smart Data Acquisition System (sDAS), designed and developed for this application, ensure the control of the whole system. After presenting the system, preliminary results obtained, in term of data acquisition, are discussed and perspectives addressed.
\end{abstract}

\section{Introduction}

Since the last evolutions of the French Thermal Regulation (Réglementation Thermique 2012 RT2012), an important gap for building energy performance requirements has been crossed. Another significant step is planned for the next Building Thermal Regulation RT2020 that will address positive energy performances as a reference for new building projects. So being able to conduct an accurate thermal estimation of building envelope performance has become crucial for building retrofitting but also to control energy efficiency of next buildings generation. Currently there is one international norm [1] that defines an in-situ measurement method of the thermal resistance of insulated building walls based on the use of contact sensors (thermocouples and fluxmeters). In the research framework of the RESBATI project, an in-situ thermal resistance estimation protocol is studied and developed, combining active thermal measurements with infrared camera, local temperature and heat flux sensors with inverse models. A smart Data Acquisition System (sDAS), designed and developed for this system, ensure the control of the whole system. Preliminary measurements are presented. They were carried out on a reduced size specimen of built at Ifsttar and tested with alternative approach using laboratory equipment [2]. Discussion on current achievement and perspectives are proposed.

\section{Instrumentation}

The smart Data Acquisition System (sDAS), designed and developed for this system, ensure the control of the whole system. Its architecture leans on three parts:

- A local instrumentation (Pt100 surface temperature, heat flux sensor, air temperature sensors and an uncooledmicrobolometer infrared camera) with their adapted signal conditioning modules (electronic designed for this application).

- An electrical regulator (PWM) connected to halogen lamp (from $50 \mathrm{~W}$ to $250 \mathrm{~W}$ ) controls the radiant heat emitted through a Fresnel lens to collimate the heat radiation toward the studied wall surface.

- A software to manage the sDAS and to estimate the thermal resistance of the wall.

The figure 1 (left) shows the electronic part of the datalogger studied and developed. This device allows managing 6 sensors for the local instrumentation Pt100 surface temperature, heat flux sensor, and air temperature sensors. The dimensions of this device are $360 \times 360 \times 180 \mathrm{~mm}^{3}$.

This datalogger is made up of 5 parts : (1) Arduino® MEGA 2560 ( 54 I/O digitals), 16 analogical inputs, (2), electronic card for the signal conditioning for both heat flux sensor, (3) et (4), a signal conditioner for 4 pt 100 et (5), general supply $230 \mathrm{AC} / \mathrm{CC}-15 \mathrm{Vcc}$.

The conditioned electrical regulator (PWM) connected to halogen lamp is shown in figure 1 (right).
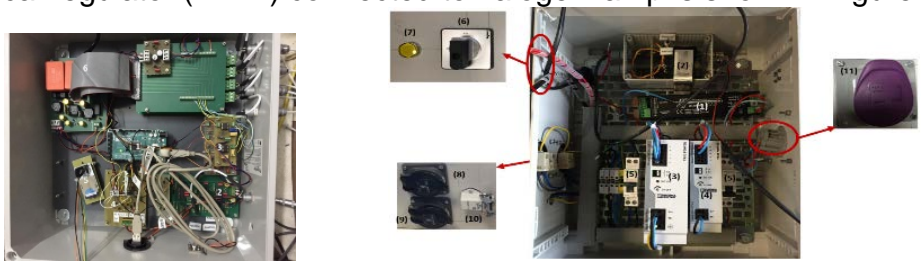

Fig. 1 Photography of the preliminary datalogger.(left) and power unit time control for halogen lamp (right) 
Figure 2 (left) shows the heating system implemented on a test bench wall developed in this work. This device is able to illuminate a wall of a building thanks to the Fresnel lens. The halogen lamp is placed on the focal length $(f=213.36 \mathrm{~mm})$ of the lens. In our case study, at this focal length, the diameter of the illuminate area is $30 \mathrm{~cm}$; to obtain an illumination area of $600 \mathrm{~mm}$, it is necessary to defocus the lamp. The electrical regulator (PWM) connected to halogen lamp (from 50 $\mathrm{W}$ to $250 \mathrm{~W}$ ) controls the radiant heat emitted through the Fresnel lens to collimate the heat radiation toward the studied wall surface. Figure 2 (right) shows an overview of the Human Machine Interface (HMI) connected to the Arduino board (ATmega2560) that ensures the control of the sDAS and also operates the measurements with the implemented local sensors.
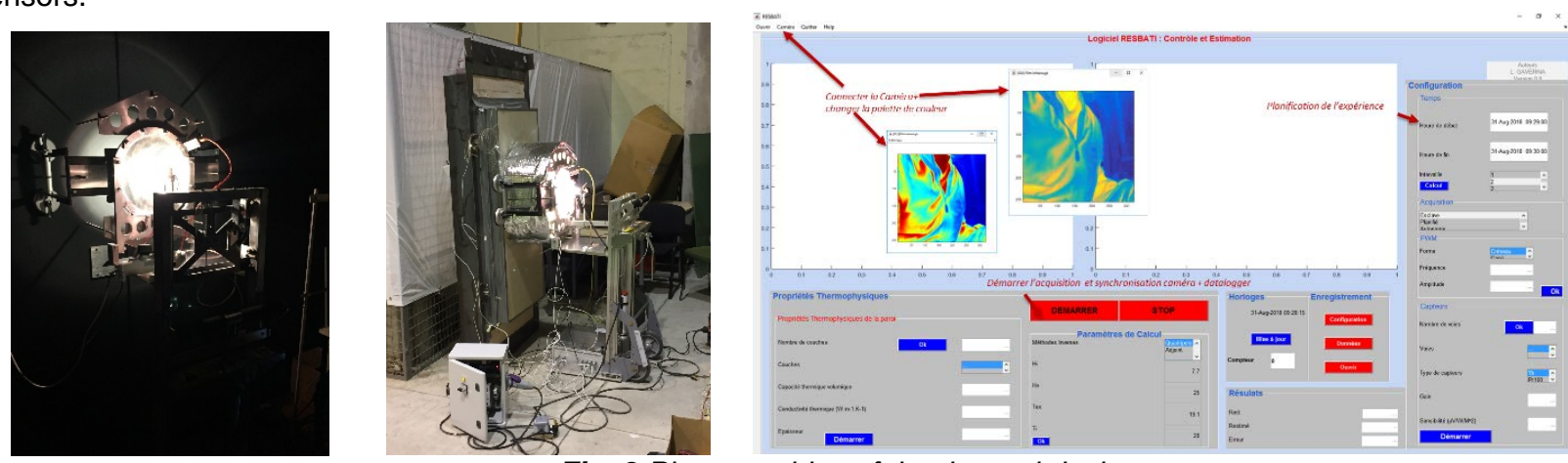

Fig. 2 Photographies of the thermal device.

\section{Preliminary results}

The device is held on the building wall by a stacker (figure 2 middle). The stacker has a head up to of $1.70 \mathrm{~m}$. The additional IR camera is fixed on the shoulder of the Fresnel lens through an adjustable support. The camera used is a FLIR ${ }^{\mathrm{TM}}$ A35 camera $(7.5-13 \mu \mathrm{m})$ with an uncooled detector of $320 \times 256$ pixels. The camera was connected through an Ethernet interface (PoE) to a portable computer (supervisor).

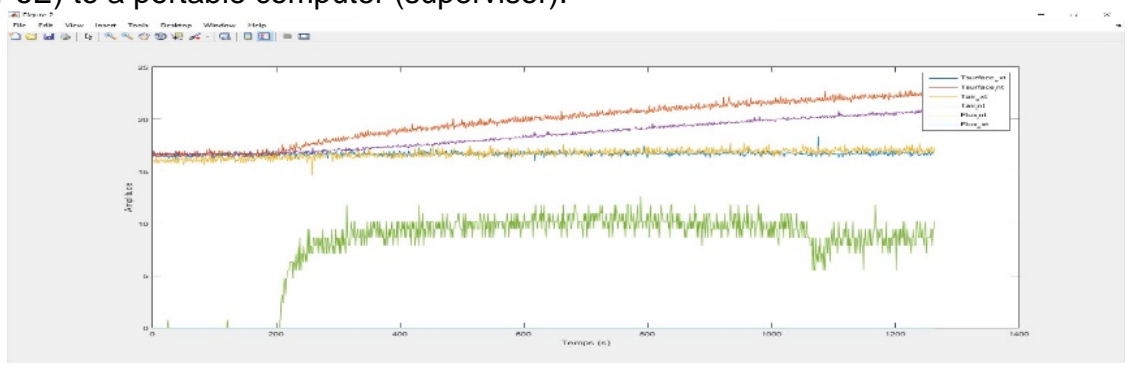

Fig. 3 Results

Figure 3 shows an example of heat flux density, air and surface temperature measurements on the heated and non-heated face of the specimen wall, for a step heating command.

\section{Conclusion and perspectives}

A full active system was studied and developed. First experiments shows promising results in term of measurement functionalities obtained with this designed system. In future works a measurements campaign will took place on a specifically designed and instrumented wall built for the project, and characterized by using standardized protocols [3]. Thermal resistance estimations will be carried out by two different inverse methods (one based on Adjoint state formulation and a second one based on thermal quadrupole formalism).

\section{Acknowledgments:}

Authors want to thank the ANR (French National Research Agency) for supporting this work through the project RESBATI under Grant agreement NANR-16-CE22-0010-02.

\section{REFERENCES}

[1] ISO 9869-1:2014 Isolation thermique - Éléments de construction - Mesurage in situ de la résistance thermique et du coefficient de transmission thermique - Partie $1:$ méthode du fluxmètre

[2] Y. Yang, T. Vogt Wu, A. Sempey, A. Sommier, J. Dumoulin and J-C. Batsale, "Short time non-destructive evaluation of thermal performances of building walls by studying transient heat transfer “, Energy and Buildings, Elsevier, Vol. 184, pp. 141-151, 2019, https://doi.org/10.1016/j.enbuild.2018.12.002 .

[3] ISO 8990 Thermal insulation -- Determination of steady-state thermal transmission properties -- Calibrated and guarded hot box. 